\title{
Comparison of eDNA and visual surveys for rare and cryptic bromeliad-dwelling frogs
}

\author{
Izabela M. Barata ${ }^{1,2,3}$, Richard A. Griffiths ${ }^{1}$, Deborah J. Fogell ${ }^{1,4}$ \& Andrew S. Buxton ${ }^{1,5}$ \\ ${ }^{1}$ Durrell Institute of Conservation and Ecology, School of Anthropology and Conservation, University of Kent, Marlowe Building, Canterbury, Kent, CT2 7NR, UK \\ ${ }^{2}$ Instituto Biotrópicos, Diamantina, Minas Gerais, Brazil \\ 3 present address: Durrell Wildlife Conservation Trust, Les Augrès Manor, Trinity, Jersey, JE3 5BP, Channel Island \\ ${ }^{4}$ Institute of Zoology, Zoological Society of London, Regents Park, London, NW1 4RY, UK \\ ${ }^{5}$ Amphibian and Reptile Conservation Trust, 744 Christchurch Road, Boscombe, Bournemouth, Dorset, BH7 6BZ, UK
}

Surveys of rare or cryptic species may miss individuals or populations that are actually present. Despite the increasing use of environmental DNA (eDNA) analysis to survey species in ponds, rivers, and lakes, very few studies have attempted to use eDNA for the detection of species using very small water bodies such as those accumulated within plants. Our aim was to investigate the feasibility of an eDNA sampling method for detecting Crossodactylodes itambe, an endemic bromeliad-dwelling frog from a remote location in Brazil. We collected water samples from 19 bromeliads for which we had observational data from direct visual surveys. We compared occupancy estimated from direct observations with the results from quantitative real-time PCR based eDNA assays. For observational surveys, we used a single season occupancy model. We applied a novel Bayesian occupancy model to estimate occupancy from eDNA samples, as well as false positives and false negatives at different stages of the workflow. eDNA from bromeliad tanks provided reliable estimates, with very low error levels and improved detection when compared to detectability from direct observation. Estimated occupancies using eDNA and visual survey methods were similar. The method is feasible for species restricted to small water bodies and exposed to direct UV radiation, and particularly useful to survey remote locations and confirm species presence. eDNA analysis provides a viable alternative to destructive sampling of bromeliads or direct observation methods that require logistically challenging repeated observations. Therefore, eDNA methods may be widely applicable to sampling programmes of other amphibians that live in plants.

Keywords: bromeliad, eDNA detection, false-positive, amphibian, occupancy, phytotelm

\section{INTRODUCTION}

S pecies surveys using direct observations suffer from the problem of individuals or populations being missed (MacKenzie et al., 2002). Such imperfect detection is caused by a wide variety of factors, including time of survey and temperature (Sewell et al., 2010), observer experience (Grant et al., 2005; Fitzpatrick et al., 2009) or simply individuals being obscured from view. When making observations, the presence of a surveyor can alter the behaviour of the target organism, reducing the likelihood of it being observed (Barata et al., 2017, 2018a). To account for imperfect detection, repeated visits are required to control for variation in detectability (MacKenzie et al., 2002). However, detection does not necessarily require direct observation. An increasing number of indirect survey methods are emerging such as environmental DNA (eDNA) analysis. Indeed, eDNA surveys can outperform direct observation surveys (Lopes et al., 2017; Burns et al., 2020), but this varies according to the ecological characteristics of the targeted organism (Takahara et al., 2019).
Surveys targeting eDNA involve the collection of environmental samples from a location which usually comprises water, soil or sediments (Turner et al., 2015; Buxton et al., 2018; Spitzen-van der Sluijs et al., 2020; Valentin et al., 2020). These samples are then processed following forensic protocols for the extraction of DNA that has been released by organisms into the environment. Where possible, the DNA that is extracted and amplified from these environmental samples is then identified to species level by comparing their sequences to a reference DNA library, thereby allowing for inferences concerning species presence in that habitat (Jane et al., 2015). Detection of species using eDNA methods has become commonplace in environments where detectability of a target species may be relatively low, such as ponds (Harper et al., 2018), lakes, rivers, and streams (Sales et al., 2019; Bedwell et al., 2020). However, very few studies have attempted to use eDNA methods for the detection of species using very small bodies of water such as aggregations of water collected within plants (also known as a phytotelm), e.g. within the tanks of bromeliads. Only two previous studies have used eDNA 
methods in the survey of bromeliad tanks (Brozio et al., 2017; Torresdal et al., 2017), both of which targeted the Critically Endangered Trinidad golden treefrog (Phytotriades auratus), an elusive species that requires destructive sampling (i.e., bromeliad destruction).

Crossodactylodes itambe is a small frog species endemic to the summit of one mountain, the Itambe summit, Minas Gerais state, in south-eastern Brazil $\left(18^{\circ} 23^{\prime} \mathrm{S}_{43^{\circ}} 20^{\prime} \mathrm{W}\right.$; datum WGS 84$)$. The species exclusively lives within ground bromeliads (Vriesea medusa), which are found on high elevation rocky outcrops (Barata et al., 2013). Crossodactylodes itambe is restricted to bromeliads at $1800 \mathrm{~m}$ above sea level or higher with a total range of less than $0.5 \mathrm{~km}^{2}$, although the plants can be found at lower altitudes (Santos et al., 2017). Species occupancy increases at higher elevation (Barata et al., 2017) and abundance of individuals is related to bromeliad structure, such as plant size and the volume of water retained by the central tank (Barata et al., 2018b). The restricted range and habitat requirements for the species make it highly vulnerable to extinction from climate change, wildfires or disease, and therefore a priority for conservation monitoring.

Due to the remote location, nocturnal activity of the species (Barata et al., 2018a) and a detection probability of 0.40-0.65 (Barata et al., 2017), visual surveys are labour intensive and costly. Power analysis conducted by Barata et al. (2017) suggests that when using visual encounters, an observer would be required to make at least three to four visits and 143 bromeliads would need to be surveyed to have an $80 \%$ chance of detecting a change of $30 \%$ in the population. Furthermore, when searching for new populations of rare and cryptic species, only a subset of the potentially highly suitable areas can be surveyed because of logistical and financial constraints (I.M. Barata, unpublished data). Consequently, it would be highly beneficial to develop a passive survey method with an equivalent or greater detectability from a single site visit than visual encounters. A passive method would provide substantial savings in terms of logistical and survey efforts, without compromising the ability to detect population changes within this highly vulnerable population or finding new populations at surrounding locations.

Here we develop species-specific PCR primers for C. itambe and test the practicalities of collecting eDNA samples from ground bromeliads in a remote location. Bromeliads in this location grow in an outcrop among shrub and herbs and are exposed to direct UV radiation, which can potentially increase DNA degradation rates (Strickler et al., 2015). We compare the known occupancy estimated from observational surveys (from multiple visits in 2014-17) with the results from quantitative realtime PCR ( $q P C R$ ) based eDNA assays. Our main goal was to test the feasibility of a passive sampling method in detecting $C$. itambe in an exposed montane area with the challenges of high UV radiation, with a view to applying the method more widely to other amphibian species that are restricted to bromeliads.

\section{METHODS}

Site selection

A tank bromeliad is a type of phytotelm that accumulates rainwater at the base of each leaf axil and in the central tank, thereby providing a microhabitat for other species (Lehtinen, 2004). We selected 19 bromeliads for eDNA sample collection, 11 of which were known to have been occupied by $C$. itambe based on previous studies and detection histories (Barata et al., 2017). The remaining eight bromeliads had no $C$. itambe recorded within them over the previous four years and were therefore considered to be unoccupied by frogs. All samples from bromeliads were from within the known range of the frog, on the Itambe summit, Minas Gerais.

Additionally, two samples were collected from flowing water in the vicinity of the study site as field negative samples. These were collected to ensure no contamination occurred in the field, as this is a potential risk when sampling in remote locations. Whilst we acknowledge that filtering sterile water on site as a field negative is a more standard approach, it was decided not to increase the volume and weight of materials needing to be carried due to the logistics of accessing the remote location. As flowing water is not used by the target species, it was highly unlikely to contain target DNA. If these results returned negative, we could be confident there was no in-field contamination.

\section{Visual encounter surveys}

Observational surveys were conducted across four years, from 2014 to 2017, during the rainy season (between October to March). Bromeliads were tagged with individual numbers that allowed repeated visits in multiple years. For each year, visual encounter surveys consisted of three to six consecutive visits to the same site to create a detection history of presence (1) and absence (0). Visual surveys were conducted at night by a team of two people, with only the most experienced observer taking notes on species presence/absence to avoid observational bias (Barata et al., 2017). Bromeliad selection for eDNA samples was based on previously known detection histories between 2014 and 2016 and an additional survey in 2017 during four consecutive nights. Surveys and detection histories from previous years provided estimates of species occupancy and detection (Barata et al., 2017). The surveys in 2017 confirmed the same species presence/absence pattern observed in previous studies and allowed the collection of water samples from selected bromeliads.

\section{Environmental DNA sample collection}

Samples were collected using syringe filtration and $0.22 \mu \mathrm{m}$ (PVDF membrane type, gamma irradiated) MilliporeTM SterivexTM filter capsules. To prevent contamination, we prepared individual sterile sample collection kits to be used at each bromeliad before conducting fieldwork. Each kit contained two pairs of gloves, a $0.22 \mu \mathrm{m}$ filter capsule, a $60 \mathrm{~mL}$ luer-lock syringe, a $30 \mathrm{~mL}$ container filled with absolute ethanol, a $10 \mathrm{ml}$ syringe with $1 / 2$ " needle, two luer-lock caps, a $50 \mathrm{~mL}$ centrifuge tube and a small zip-lock bag (Fig. 1A). In the 
field, water was drawn from either the centre or lateral leaves of the bromeliad using the sterile $60 \mathrm{~mL}$ luer-lock syringe (Fig. 1B); the filter capsule was attached to this syringe which was used to push the water across the filter membrane (Fig. 1C). This procedure was repeated twice to filter a total of $120 \mathrm{~mL}$ of water. Once the whole water sample was filtered or the capsule had become blocked (which was the case for one sample), $10 \mathrm{~mL}$ of absolute ethanol was added to each filter as a preservative using the $10 \mathrm{~mL}$ syringe and $1 / 2$ " needle. Each capsule was sealed with luer-lock caps, re-sealed in an individual 50 $\mathrm{mL}$ centrifuge tube and stored in a sample bag to prevent contamination while in storage and transport (Fig. 1D). We numbered each sample (filters, containers and bags) with the reference number of the bromeliad from individual tags.
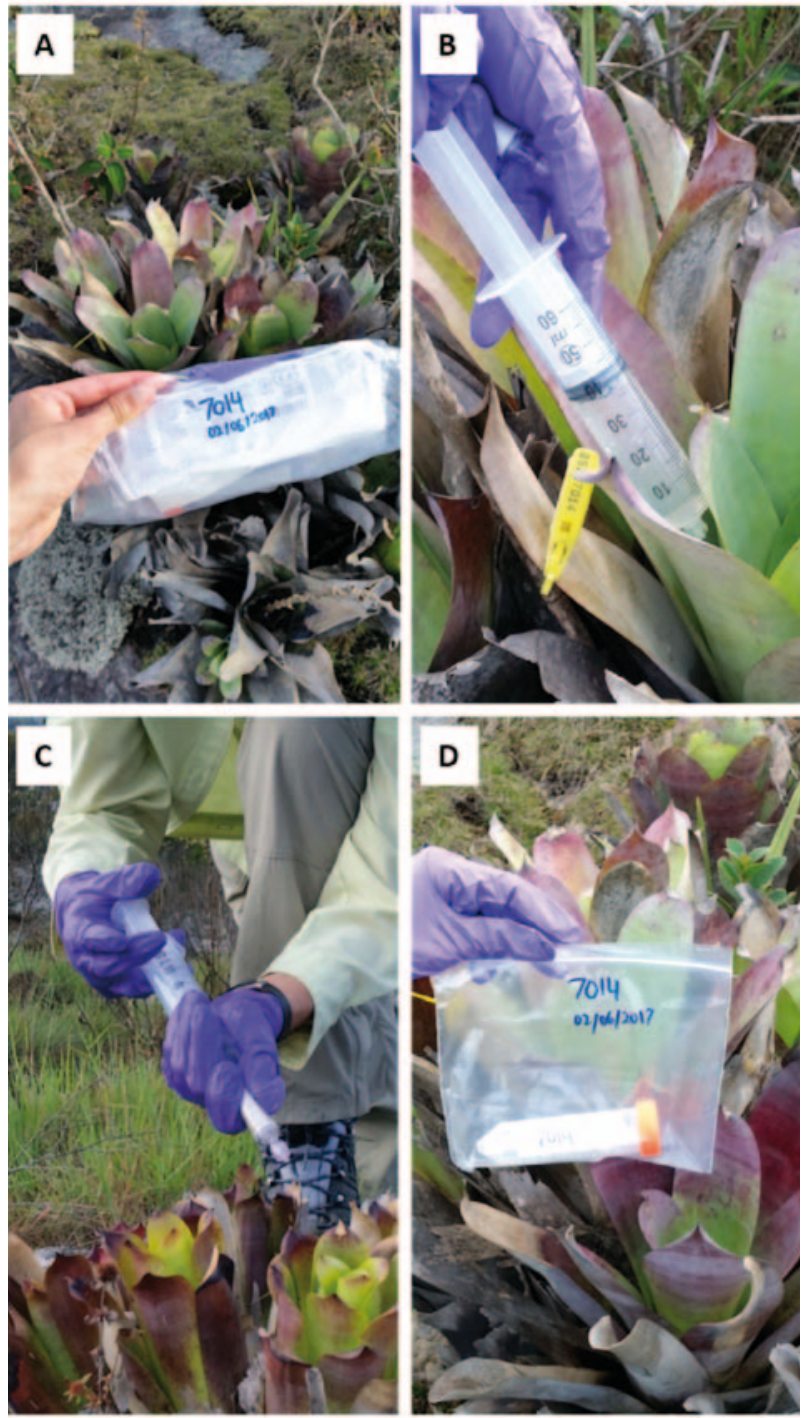

Figure 1. Environmental DNA sample collection demonstration: A) sample collection kit; B) water collection from lateral leaves of the bromeliaed using a sterile $60 \mathrm{~mL}$ syringe; C) pushing the water across the $0.22 \mu \mathrm{m}$ filter capsule; and D) individual capsule sealed in a tube and stored in a sample bag to prevent contamination.

\section{eDNA extraction}

DNA extraction was undertaken in a dedicated lab, within a UV hood. All equipment and work stations were sterilised using a combination of $10 \%$ bleach solution and/or UV light in advance of use. Standard laboratory protective equipment was worn at all times. DNA extraction followed a modified Qiagen ${ }^{\circledR}$ DNeasy ${ }^{\circledR}$ blood and tissue kit protocol, adapted from Spens et al. (2016). The ethanol preservative was removed from the MilliporeTM SterivexTM filter capsule by attaching a sterile syringe and passing air across the capsule, collecting the liquid in a $2 \mathrm{~mL}$ micro-centrifuge tube. $50 \mathrm{uL}$ of $3 \mathrm{M}$ sodium acetate solution per millilitre of ethanol recovered was added, these samples were then incubated at $-80{ }^{\circ} \mathrm{C}$ for 10 minutes to aid in DNA precipitation before being centrifuged at 14000 RPM for 15 minutes to collect any precipitate as a pellet on the side of the tube with supernatant discarded. $180 \mu \mathrm{L}$ of ATL buffer and $20 \mu \mathrm{L}$ of proteinase $\mathrm{K}$ from the extraction kit was added to each micro-centrifuge tube, which was then vortexed for 15 seconds to suspend the pellet and mix. Samples were incubated on a rotating block at $56{ }^{\circ} \mathrm{C}$ overnight. AL buffer and ice-cold absolute ethanol was then added to each micro-centrifuge tube in a 1:1:1 ratio with the incubated contents of the tube.

$720 \mu \mathrm{L}$ ATL buffer and $80 \mu \mathrm{L}$ of proteinase $\mathrm{K}$ from the extraction kit were added directly into each of the filter capsules, the caps replaced, and filter units sealed with Parafilm ${ }^{\circledR}$, and incubated at $56^{\circ} \mathrm{C}$ overnight on a rotating block. The liquid was removed from the filter using a sterile syringe and by passing air through the capsule, collecting the buffer in a fresh micro-centrifuge tubes. AL buffer from the extraction kit and ice-cold ethanol were then added in a 1:1:1 ratio (samples were split across two tubes per capsule to accommodate the volume). Product extracted from the ethanol preservation buffer and the filter capsule for each sample were then combined, passing both across the same mini-spin column from the extraction kit. Extraction continued as per extraction kit manufacturer's protocol eluting into $200 \mu \mathrm{L}$ of warm $A E$ buffer.

\section{Primer development}

Primers suitable for use with eDNA were developed to amplify a short region of the Cytochrome Oxidase 1 gene (COI) of $C$. itambe, based on the sequence identified by Santos et al. (2017); NCBI accession number KY362551. Primers were designed using the program Primer 3 (Koressaar \& Remm, 2007; Untergasser et al., 2012; Koressaar et al., 2018). Conditions were set to identify primers between 18 and 23 base pairs in length to amplify a region between 75 and 100 base pairs long. We specified that no runs of greater than three base pairs should be included, with a GC content of between 40 and $50 \%$, and an optimum melt temperature of $60{ }^{\circ} \mathrm{C}$. A set of primers was identified to amplify an 83 base pair sequence (Table 1 ). The primer sequences were tested in silico with a NCBI blast search to check for cross amplification with other species. No species were found to have a $85 \%$ or greater match to either the forward or reverse primer. Additionally, whilst other 
frogs (Bokermannohyla nanuzae) were occasionally seen using the bromeliads in the study area over this 4-year period (Barata I.M., personal observation), no other amphibian species using the bromeliads were recorded during the observational surveys (i.e., the night before eDNA samples were taken). Primers could not be tested in vitro due to the difficulties in obtaining and exporting tissue samples of the relevant species.

Table 1. Primer sequences generated using Primer 3 for detection of $C$. itambe from eDNA.

\begin{tabular}{ccccc}
\hline $\begin{array}{c}\text { Primer } \\
\text { name }\end{array}$ & Length & $\begin{array}{c}\text { Melting } \\
\text { temperature }\end{array}$ & GC\% & Sequence \\
\hline CICO1-F & 20 & 59.78 & 50 & tacttgcttctgctggcgta \\
CICO1-R & 20 & 59.59 & 55 & $\begin{array}{c}\text { ggcatgggctaagt- } \\
\text { taccag }\end{array}$ \\
\hline
\end{tabular}

\section{qPCR}

qPCR was conducted in a separate room to DNA extraction. All equipment and work surfaces were sterilised using either a $10 \%$ bleach solution and/or UV light in advance of use and appropriate personal protective equipment was worn. Plate set-up was conducted in a UV hood dedicated to low concentration DNA work. qPCR was performed using a SYBR Green assay, with eight replicates per sample. The qPCR amplification procedure consisted of $10 \mu \mathrm{L}$ of Applied Biosystems ${ }^{\mathrm{TM}}$ PowerUp ${ }^{\mathrm{TM}}$ SYBR $^{\mathrm{TM}}$ Green Master Mix, $2 \mu \mathrm{L}$ of each primer at a concentration of 10 $\mu \mathrm{M} / \mu \mathrm{L}$, and $4 \mu \mathrm{L}$ of template DNA, in a final reaction volume of $20 \mu \mathrm{L}$. qPCR conditions consisted of two activation steps at $50{ }^{\circ} \mathrm{C}$ for 2 minutes followed by 95 ${ }^{\circ} \mathrm{C}$ for 2 minutes, then 40 cycles of $95{ }^{\circ} \mathrm{C}$ for 15 seconds, $59{ }^{\circ} \mathrm{C}$ for 15 seconds and $72{ }^{\circ} \mathrm{C}$ for 1 minute. A melt curve was then performed ramping up from $55{ }^{\circ} \mathrm{C}$ to 95 ${ }^{\circ} \mathrm{C}$ in $0.5{ }^{\circ} \mathrm{C}$ increments. Three negative control samples of $\mathrm{ddH}_{2} \mathrm{O}$ were included in each qPCR run, to check for contamination. A replicate was classed as positive when an exponential growth phase in relative florescence was identified and the melt curve indicated a temperature of between $82{ }^{\circ} \mathrm{C}$ and $82.5^{\circ} \mathrm{C}$, indicating the fluorescence was not caused by primer dimer.

Following qPCR analysis, each sample was checked for inhibition by adding a known quantity of non-target DNA to each sample. The assay consisted of $10 \mu \mathrm{L}$ of Applied Biosystems $^{\mathrm{TM}}$ PowerUp ${ }^{\mathrm{TM}}$ SYBR ${ }^{\mathrm{TM}}$ Green Master Mix, 4 $\mu \mathrm{L}$ of eDNA sample, $2 \mu \mathrm{L}$ of a tissue extract from great crested newts (Triturus cristatus), $2 \mu \mathrm{L}$ of forward and reverse primers for our targeted species ( $C$. itambe), as well as the forward and reverse primers for great crested newts (TCCBL and TCCBR; Thomsen et al., 2012) at a concentration of $10 \mu \mathrm{M} / \mu \mathrm{L}$, in a final reaction volume of $20 \mu \mathrm{L}$. The $\mathrm{qPCR}$ and melt curve conditions replicated those stated above, with two negative control samples included in each qPCR run. Samples failing to amplify our target species' DNA or amplifying more than 1 cycle later than control samples were considered to contain PCR inhibitors.

\section{Data analysis for species detection}

We used a single-season occupancy model (MacKenzie et al., 2002) to estimate detection and occupancy rates from the observational data collected in the field. We used previously published detection histories (Barata et al., 2017) and included only bromeliads for which we had eDNA samples. Although occupancy models can accommodate covariates that explain both parameters (MacKenzie et al., 2002), we opted to run a null model (i.e., with no covariates). As our aim was to obtain overall estimates for comparisons without exploring covariates; given the small size of our dataset, we wanted to avoid overparameterisation of the model. We controlled for variation in detection in our data by using observations from a single experienced observer (Barata et al., 2017). We used a free online tool to analyse qPCR based eDNA data to generate occupancy and detectability information (https://blogs.kent.ac.uk/edna). This tool is based on Griffin et al. (2020) and uses a Bayesian framework to identify: the probability of species occupancy; stage 1 (the sample collection phase) true and false positive rates; and stage 2 (the laboratory phase) true and false positive rates. Stage 1 true positive rate $(\theta 11)$ is the probability that a water sample collected from an occupied site includes DNA of the target species, with stage 1 false negative rate being the complement of this. Stage 1 false positive rate $(\theta 10)$ is the probability that a water sample collected from an unoccupied site includes DNA of the target species. Stage 2 true positive rate $(p 11)$ is that an individual PCR replicate of a sample containing target DNA is positive, with stage 2 false negative being the complement of this. Stage 2 false positive rate $(p 10)$ is the probability that an individual PCR replicate of a sample that does not include DNA of the target species returns amplification. These differ from standard observational occupancy models as eDNA sampling is a two-phase process with potential for error to be introduced at both sample collection and sample analysis phases. Conversely, direct observation has a single phase where the species is either observed or not observed.

\section{RESULTS}

No target DNA was amplified from field or laboratory negative control samples by 40 qPCR cycles, and no sample demonstrated characteristics that indicated PCR inhibitors were present. All 11 samples collected from bromeliads with known species presence amplified target DNA, of which 10 showed amplification in all eight qPCR replicates, with the remaining sample showing amplification in seven of the eight qPCR replicates (Table 2). Additionally, one sample from a bromeliad with no known occupancy showed amplification in a single qPCR replicate (bromeliad 8125; Table 2). One sample from a previously unoccupied bromeliad had amplification in five of the eight replicates (bromeliad 7007; Table 2). However, this filter was damaged and leaked during transportation, possibly leading to contamination and a false positive. Therefore, we excluded this result from any further data analysis. These two eDNA positive but 
Table 2. Water samples collected in the field for 19 bromeliads and two field negatives (FN) with sample number, characteristics of bromeliads (elevation given in metres above sea level, size given as bromeliad height in centimetres), and results from direct observations detection history $(0=$ absence and $1=$ presence) and environmental DNA (eDNA) analysis ( $\mathrm{P}=$ positive and $\mathrm{N}=$ Negative).

\begin{tabular}{|c|c|c|c|c|c|c|c|c|c|}
\hline \multirow{2}{*}{ Sample number } & \multicolumn{2}{|c|}{ Bromeliad } & \multicolumn{4}{|c|}{ Direct observation } & \multirow{2}{*}{ eDNA } & \multirow{2}{*}{$\begin{array}{l}\text { eDNA positive } \\
\text { replicates }\end{array}$} & \multirow{2}{*}{$\begin{array}{r}\text { Signs of } \\
\text { inhibitior }\end{array}$} \\
\hline & Elevation & Size & 2014 & 2015 & 2016 & 2017 & & & \\
\hline 8131 & 2063.4 & 63 & 0 & 1 & 1 & 1 & $P$ & $7 / 8$ & $N$ \\
\hline 6940 & 2047.6 & 52 & 1 & 1 & 1 & 1 & $P$ & $8 / 8$ & $N$ \\
\hline 1149 & 2013.7 & 61 & 0 & 0 & 1 & 1 & $P$ & $8 / 8$ & $\mathrm{~N}$ \\
\hline 6950 & 1987.1 & 44 & 1 & 1 & 1 & 1 & $\mathrm{P}$ & $8 / 8$ & $\mathrm{~N}$ \\
\hline 6983 & 2029.6 & 40 & 1 & 1 & 0 & 1 & $P$ & $8 / 8$ & $\mathrm{~N}$ \\
\hline 7015 & 1934.3 & 78 & 0 & 0 & 1 & 1 & $P$ & $8 / 8$ & $\mathrm{~N}$ \\
\hline 2222 & 1885.9 & 56 & 1 & 1 & 1 & 1 & $P$ & $8 / 8$ & $\mathrm{~N}$ \\
\hline 6963 & 1874.8 & 48 & 0 & 1 & 1 & 1 & $P$ & $8 / 8$ & $\mathrm{~N}$ \\
\hline 6994 & 1873.8 & 44 & 1 & 1 & 1 & 1 & $P$ & $8 / 8$ & $\mathrm{~N}$ \\
\hline 2802 & 1911.5 & - & - & - & - & 1 & $P$ & $8 / 8$ & $\mathrm{~N}$ \\
\hline 8161 & 1769.4 & 53 & 0 & 0 & 1 & 1 & $\mathrm{P}$ & $8 / 8$ & $\mathrm{~N}$ \\
\hline 7007 & 2039.9 & 52 & 0 & 0 & 0 & 0 & $P$ & $5 / 8$ & $\mathrm{~N}$ \\
\hline 8125 & 1713.1 & 69 & 0 & 0 & 0 & 0 & $P$ & $1 / 8$ & $N$ \\
\hline 2168 & 2024.8 & 43 & 0 & 1 & 0 & 0 & $\mathrm{~N}$ & $0 / 8$ & $\mathrm{~N}$ \\
\hline 6929 & 1927.7 & 52 & 0 & 0 & 0 & 0 & $N$ & $0 / 8$ & $\mathrm{~N}$ \\
\hline 7014 & 1920.2 & 65 & 0 & 0 & 0 & 0 & $N$ & $0 / 8$ & $\mathrm{~N}$ \\
\hline 8070 & 1771.8 & 56 & 0 & 0 & 0 & 0 & $N$ & $0 / 8$ & $\mathrm{~N}$ \\
\hline 8164 & 1841.7 & 62 & 0 & 0 & 0 & 0 & $\mathrm{~N}$ & $0 / 8$ & $\mathrm{~N}$ \\
\hline 6981 & 1716.6 & 62 & 0 & 0 & 0 & 0 & $\mathrm{~N}$ & $0 / 8$ & $N$ \\
\hline FN 1 & 1597.1 & - & - & - & - & 0 & $\mathrm{~N}$ & $0 / 8$ & $\mathrm{~N}$ \\
\hline FN 2 & 1558.9 & - & - & - & - & 0 & $\mathrm{~N}$ & $0 / 8$ & $N$ \\
\hline
\end{tabular}

Table 3. Parameter estimates from occupancy models derived from direct observational surveys and eDNA samples with multiple qPCR replicates (for observational surveys using occupancy models, $\mathrm{Cl}$ = confidence interval; for eDNA samples using Bayesian framework, $\mathrm{Cl}=$ credible intervals).

\begin{tabular}{|c|c|c|c|c|}
\hline \multirow[b]{2}{*}{ Parameter } & \multirow[b]{2}{*}{ Estimate } & \multicolumn{2}{|c|}{$\mathrm{Cl}$} & \multirow[b]{2}{*}{ Description } \\
\hline & & Upper & Lower & \\
\hline \multicolumn{5}{|c|}{ Observational surveys } \\
\hline$\Psi$ & 0.61 & 0.38 & 0.80 & Occupancy estimated from observational survey data \\
\hline $\mathrm{p}$ & 0.77 & 0.62 & 0.88 & Detection probability for visual night encounters \\
\hline \multicolumn{5}{|l|}{ eDNA sample } \\
\hline$\Psi$ & 0.61 & 0.38 & 0.81 & Occupancy estimated from eDNA samples \\
\hline$\theta 10$ & 0.04 & 0.00 & 0.20 & Stage 1 (sample collection) false positive rate \\
\hline$\theta 11$ & 0.97 & 0.85 & 1.00 & Stage 1 true positive rate \\
\hline $1-\theta 11$ & 0.03 & & & Stage 1 false negative rate (given by $1-\theta 11$ ) \\
\hline p10 & 0.02 & 0.00 & 0.07 & Stage 2 (laboratory analysis) false positive rate \\
\hline p11 & 0.98 & 0.94 & 1.00 & Stage 2 true positive rate \\
\hline 1-p11 & 0.02 & & & Stage 2 false negative rate (given by 1-p11) \\
\hline
\end{tabular}

observationally negative bromeliads were both found within the existing known range of the species. Overall, we had a naïve occupancy rate of $66.7 \%$ using the eDNA method (12 positives out of 18 samples), compared to $61.1 \%$ for direct observational surveys (11 positives out of 18 surveyed sites).

From direct observational survey data, the occupancy rate was estimated to be 0.61 and detectability was 0.77 (Table 3). From eDNA analysis, a bromeliad occupancy rate of 0.61 was estimated. A false positive rate at sample collection $(\theta 10)$ of 0.04 was found, with a true positive rate at sample collection $(\theta 11)$ of 0.97 , equivalent to a $3 \%$ false negative rate (Table 3 ). This compared to the false positive rate at the laboratory analysis stage (p10) of 0.02 and true positive rate at the laboratory analysis stage ( $p 11$ ) of 0.98 , equivalent to a $2 \%$ false negative rate (Table 3 ). The conditional probability of detection analysis given by the number of amplified qPCR replicates (Fig. 2) shows the probability that an occupied site is wrongly classified as unoccupied in relation to the 
number of samples which amplify. Our results suggest a high probability of false positive detection for any sample amplifying with fewer than three positive qPCR replicates. Additionally, when five or more of the eight replicates amplify, we can be confident that the site is indeed occupied. We also observed that there is little gain in occupancy estimate with this additional effort (Fig. 2).

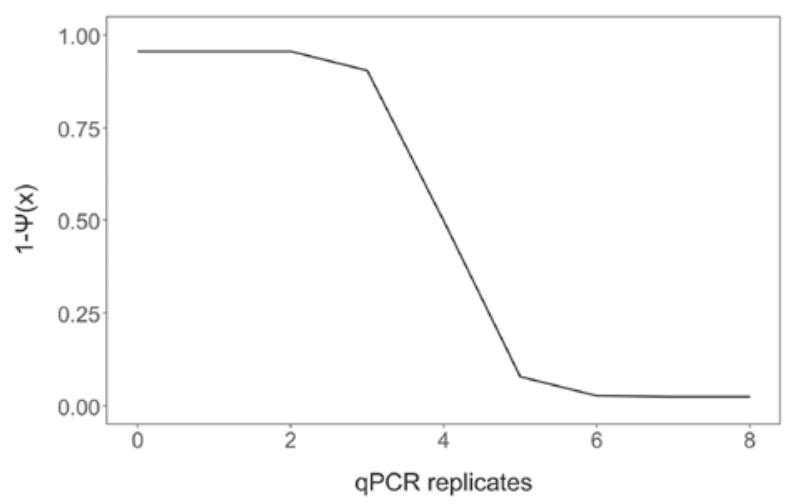

Figure 2. Posterior conditional probabilities of species absence $(1-\Psi(x))$ given by the number of amplifying qPCR replicates.

\section{DISCUSSION}

We found that eDNA from bromeliad tanks, including sample collection (stage 1) and laboratory analysis (stage 2 ), is highly reliable, with very low levels of error for both false negatives and false positives (false negative: stage 1 = $3 \%$; stage 2 = $2 \%$; false positive: stage $1=4 \%$; stage 2 $=2 \%)$. Higher error rates were observed for commercial eDNA surveys for great crested newts in ponds within the UK, from both sample collection (stage 1: false positive rate $=15 \%$; false negative rate $=27 \%$ ) and laboratory analysis (stage 2 : false positive $=5 \%$; false negative $=$ 19\%) (Griffin et al., 2020). Phytotelm-breeding species are often elusive and difficult to detect using visual surveys, and occasionally require destructive sampling methods such as removal of plants (Brozio et al., 2017; Torresdal et al., 2017). In these cases, eDNA is a reliable non-invasive method that detects species presence with very low error rates (i.e., low false positive/negative rates). With a single set of eDNA samples we were able to accurately detect species presence in every bromeliad confirmed to be occupied through repeated direct observations, as well as in one bromeliad where species occupancy had not been identified. However, this additional detection had only a single positive qPCR replicate which is highly likely a case of false positive detection, as indicated by the conditional probabilities analysis.

We have demonstrated the feasibility of collecting eDNA samples in a remote setting and transporting them to a laboratory for analysis. Despite one sample being damaged in transit and the potential false positive result described above, it was possible to maintain a contamination-free environment during sample collection and transport as demonstrated by the absence of amplification in the samples of water collected from streams close to the study site. It is also evident from the high proportion of qPCR replicates amplifying in the confirmed positive samples that for a species which spends a large part of its life cycle within the phytotelm (such as a bromeliad), sampling only a small volume of water is not a limiting factor for the recovery of target DNA. Whilst we present the results from a relatively small sample size, we successfully demonstrate the feasibility of extracting and amplifying DNA from water samples as little as $120 \mathrm{ml}$. The conditional probability of species detection analysis showed that above five qPCR positive replicates, there is limited gain in the estimate of occupancy. Therefore, the number of qPCR replicates may be excessive and a reduction in laboratory replication may be possible without reducing the occupancy estimate; however further analysis would need to be undertaken to confirm this observation which would require a larger data set.

A major advantage of the use of eDNA methods in remote locations is that it can reliably confirm the presence of species in bromeliads, even when bromeliads have high UV exposure, such as at the mountaintop described here. In this scenario, eDNA surveys would have wide-ranging benefits compared to multiple observational visits. Firstly, it is challenging and costly to access remote areas, requiring an experienced team of observers within an expedition that lasts a number of days. Secondly, our described method reduces bias caused by variation in observer experience (Barata et al., 2017) and is sensitive to different life stages (e.g., eggs and larvae; Zinger et al., 2020) that can be missed during visual surveys. Thirdly, eDNA is a non-invasive method that can reduce environmental impact associated with direct observations (Brozio et al., 2017; Torresdal et al., 2017). Most importantly, because high prevalence of chytrid fungus can be found in bromeliads occupied by frogs (Ruano-Fajardo et al., 2016), reduced visits could also decrease the potential risks associated with the spread of wildlife pathogens by the survey team, while eDNA samples can also be reanalysed for the pathogen (Schmidt et al., 2013).

Despite the very high detection rates from eDNA samples, estimated occupancies using eDNA and direct observations were similar. This has implications for the use of eDNA methods for occupancy monitoring since eDNA analysis has laboratory and consumable costs above those incurred in direct observational surveys. For monitoring purposes, estimates of species occupancy can be available from a detection history, which would require multiple visits, and/or eDNA samples with laboratory replication. For our target species, an increase in detection does not improve statistical power and four visits are required to detect a change of $30 \%$ in the population using direct observation (Barata et al., 2017). In our case study, the mean costs per bromeliad sampled by non-invasive observational surveys (four nights with a team of two people, 21 bromeliads $=£ 21.29$ per bromeliad) is lower than the costs per sample for a single set of eDNA analysis (a one-day visit by one person, two days of lab work by one person, and laboratory supplies, 
21 bromeliads $=£ 54.92$ per bromeliad). Nonetheless, in cases where destructive sampling is required, the higher financial costs of eDNA methods could be outweighed by the costs to biodiversity conservation for habitat integrity. Therefore, a case-by-case cost-benefit analysis is recommended to ascertain whether the cost of running a full set of eDNA surveys offsets the cost of the observational survey visits.

A number of factors may influence the persistence of eDNA within the bromeliad phytotelm. Firstly, the ratio of target species biomass to available water volume in the phytotelm may influence the concentration of DNA in the water and therefore the sample. The ratio of the biomass of the species per unit volume of the water within the phytotelm will be high compared to other eDNA studies investigating species in more typical ponds, lakes and streams. Also, the volume of water retained in a bromeliad varies with the shape and size of a plant, reaching up to $2 \mathrm{~L}$ of water per plant in dry environments (Cogliatti-Carvalho et al., 2010). Considering C. itambe measures up to $18 \mathrm{~mm}$ in length with a mass of about $2 \mathrm{~g}$, this would mean a biomass to water ratio of 1:1000 for one individual per plant. This is a much higher ratio than that encountered in eDNA surveys for larger amphibians in ponds or lakes (e.g. 10 g great crested newts in a $600 \mathrm{~m}^{2}$ pond area (Jehle et al., 2011; Oldham et al., 2000), where a conservative biomass to water ratio would be $1: 1,000,000$ for 50 individuals, in a 500,000 L pond). This high ratio may contribute to the high amplification rate in positive samples, with most positive samples showing either seven or eight positive qPCR replicates.

Secondly, the degree to which the species is dependent on the phytotelm can influence DNA release into the water. If the species resides permanently in the bromeliad, more DNA may be released into the water than if it was a temporary visitor. Equally, the concentration of DNA within a sample may influence the chance of detecting the target DNA. Experimental studies could explore the relationship between persistence of eDNA in a phytotelm after introducing and removing individuals from water tanks to define an optimum time to detection. Finally, for pond breeding amphibians, surveys using eDNA methods can also account for a wide range of covariates associated with both the pond and the target species (Barnes et al., 2014), such as substrate type (Buxton et al., 2018) and seasonality (Buxton et al., 2017). For bullfrog (Lithobates catesbeianus) tadpoles in a controlled environment, degradation rates were lowest under low UV-B radiation and cold temperatures (Strickler et al., 2015). In a phytotelm, these external factors are likely to include seasonality, temperature and UV radiation exposure, particularly at high altitudes where UV rates are higher than at sea level.

We conclude that there are numerous advantages in the use of eDNA to survey cryptic species in remote locations, and to identify species presence with high detectability and low error rates. Factors influencing persistence of eDNA in small water bodies need to be further investigated to fully understand the challenges and limitations of applying eDNA methods within a phytotelm environment. The method has potential to uncover the extent of the distribution of many elusive phytotelm-breeding species with reduced expedition costs and environmental impacts. eDNA surveys are considered a promising method for amphibian monitoring regardless of species rarity (Burns et al., 2020) or population density (Lopes et al., 2017). However, the use of eDNA method for monitoring occupancy of phytotelm-dependent species will likely improve as the method becomes more cost-effective and we have a better understanding of the factors affecting detection probability in such environments. Our conclusions are applicable to other phytotelm-dependent species, but the feasibility of the method could vary with species' life history and the volume of water available for analysis.

\section{ACKNOWLEDGEMENTS}

We would like to thank the Rufford Foundation for supporting fieldwork and laboratory analysis (212642). Relevant permits were obtained by IMB (48859-4, 104/2017). IMB was supported by PhD scholarship from CAPES Foundation (BEX 13153-13-7). We are grateful for the logistical support provided by the protected area manager and park rangers from Pico do Itambe State Park during field work, and to Professor Jim Groombridge and the School of Anthropology and Conservation for provision of laboratory facilities. We are grateful to $\mathrm{Dr}$ Naiara Sales and Dr Lynsey Harper for their valuable reviews which greatly improved the quality of this manuscript.

\section{REFERENCES}

Barata, I. M., Santos, M. T. T., Leite, F. S. F. \& Garcia, P. C. A. (2013). A new species of Crossodactylodes (Anura: Leptodactylidae) from Minas Gerais, Brazil: First record of genus within the Espinhaço Mountain Range. Zootaxa 3731, 552-560.

Barata, I. M., Griffiths, R. A. \& Ridout, M. S. (2017). The power of monitoring: optimizing survey designs to detect occupancy changes in a rare amphibian population. Scientific Reports 7, 16491.

Barata, I. M., Griffiths, R. A. \& Ferreira, G. B. (2018a). Activity pattern and behavior of an endemic bromeliad frog observed through camera trapping. Herpetological Review 49, 432-438.

Barata, I.M., Silva, E.P. \& Griffiths, R.A. (2018b). Predictors of abundance of a rare bromeliad-dwelling frog (Crossodactylodes itambe) in the Espinhaço Mountain Range of Brazil. Journal of Herpetology 52, 321-326.

Barnes, M. A., Turner, C. R., Jerde, C. L., Renshaw, M. A., Chadderton, W. L. \& Lodge, D. M. (2014). Environmental conditions influence eDNA persistence in aquatic systems. Environmental Science \& Technology 48, 1819-1827.

Bedwell, M.E. \& Goldberg, C. S. (2020). Spatial and temporal patterns of environmental DNA detection to inform sampling protocols in lentic and lotic systems. Ecology and Evolution 10, 1602-1612.

Brozio, S., Manson, C., Gourevitch, E., Burns, T. J., Greener, M. S., Downie, J. R. \& Hoskisson, P. A. (2017). Development and application of an eDNA method to detect the critically endangered Trinidad golden tree frog (Phytotriades auratus) 
in bromeliad phytotelmata. PLOS ONE 12, e0170619.

Burns, T. J., Clemann, N., Rooyen, A. R. van, Scheele, B. C., Weeks, A. R. \& Driscoll, D. A. (2020). Environmental DNA sampling in a terrestrial environment: methods to detect a critically endangered frog and a global pathogen. bioRxiv preprint doi: https://doi.org/10.1101/2020.03.01.968693.

Buxton, A. S., Groombridge, J. J., Zakaria, N. B. \& Griffiths, R. A. (2017). Seasonal variation in environmental DNA in relation to population size and environmental factors. Scientific Reports 7, 1-9.

Buxton, A. S., Groombridge, J. J. \& Griffiths, R. A. (2018) Seasonal variation in environmental DNA detection in sediment and water samples. PLOS ONE 13, e0191737.

Cogliatti-Carvalho, L., Rocha-Pessôa, T. C., Nunes-freitas, A. F. \& Rocha, C. F. D. (2010). Volume de água armazenado no tanque de bromélias, em restingas da costa brasileira. Acta Botanica Brasilica 24, 84-95.

Fitzpatrick, M. C., Preisser, E. L., Ellison, A. M. \& Elkinton, J. S. (2009). Observer bias and the detection of low-density populations. Ecological Applications 19, 1673-1679.

Grant, E. H. C., Jung, R. E., Nichols, J. D. \& Hines, J. E. (2005). Double-observer approach to estimating egg mass abundance of pond-breeding amphibians. Wetlands Ecology and Management 13, 305-320.

Griffin, J. E., Matechou, E., Buxton, A. S., Bormpoudakis, D. \& Griffiths, R. A. (2020). Modelling environmental DNA data; Bayesian variable selection accounting for false positive and false negative errors. Journal of the Royal Statistical Society: Applied Statistics 69, 377-392.

Harper, L. R., Buxton, A. S., Rees, H. C., Bruce, K., Brys, R., Halfmaerten, D., Read, D. S., Watson, H. V., Sayer, C. D., Jones, E. P. \& Priestley, V. (2018). Prospects and challenges of environmental DNA (eDNA) monitoring in freshwater ponds. Hydrobiologia 826(1), 25-41.

Jane, S. F., Wilcox, T. M., Mckelvey, K. S., Young, M. K., Schwartz, M. K., Lowe, W. H., Letcher, B. H. \& Whiteley, A. R. (2015). Distance, flow and PCR inhibition: eDNA dynamics in two headwater streams. Molecular Ecology Resources 15, 216 227.

Jehle, R., Thiesmeier, B. \& Foster, J. (2011). The crested newt - a dwindling pond-dweller. Bielefield: Laurenti-Verlag.

Koressaar, T. \& Remm, M. (2007). Enhancements and modifications of primer design program Primer3. Bioinformatics 23(10), 1289-91.

Koressaar, T., Lepamets, M., Kaplinski, L., Raime, K., Andreson, R. \& Remm, M. (2018). Primer3_masker: integrating masking of template sequence with primer design software. Bioinformatics 34, 1937-1938.

Lehtinen, R. (2004). Ecology and evolution of phytotelm-breeding anurans. Miscellaneous Publications of the University of Michigan.

Lopes, C. M., Sasso, T., Valentini, A., Dejean, T., Martins, M., Zamudio, K. R. \& Haddad, C. F. B. (2017). eDNA metabarcoding: a promising method for anuran surveys in highly diverse tropical forests. Molecular Ecology Resources 17, 904-914.

MacKenzie, D. I., Nichols, J. D., Lachman, G. B., Droege, S., Andrew, J. \& Langtimm, C. A. (2002). Estimating site occupancy rates when detection probabilities are less than one. Ecology 83, 2248-2255.

Oldham, R. S., Keeble, J., Swan, M. J. S. \& Jeffcote, M. (2000).
Evaluating the suitability of habitat for the great crested newt (Triturus cristatus). Herpetological Journal 10, 143155.

Ruano-Fajardo, G., Toledo, L. F. \& Mott, T. (2016). Jumping into a trap: High prevalence of chytrid fungus in the preferred microhabitats of a bromeliad-specialist frog. Diseases of Aquatic Organisms 121, 223-232.

Sales, N. G., Wangensteen, O. S., Carvalho, D. C. \& Mariani, S. (2019). Influence of preservation methods, sample medium and sampling time on eDNA recovery in a neotropical river. Environmental DNA 1, 119-130.

Santos, M. T. T., Pezzuti, T. L., Barata, I. M., Leite, F. S. F. \& Garcia, P. C. A. (2017). The tadpole of the microendemic, bromeligenous Crossodactylodes itambe (Anura, Leptodactylidae) from the endangered 'campo rupestre' of Southeastern Brazil, with additional comments on natural history. South American Journal of Herpetology 12, 14-23.

Schmidt, B. R., Kéry, M., Ursenbacher, S., Hyman, O. J. \& Collins, J. P. (2013). Site occupancy models in the analysis of environmental DNA presence/absence surveys: A case study of an emerging amphibian pathogen. Methods in Ecology and Evolution 4, 646-653.

Sewell, D., Beebee, T. J. C. \& Griffiths, R. A. (2010). Optimising biodiversity assessments by volunteers: The application of occupancy modelling to large-scale amphibian surveys. Biological Conservation 143, 2102-2110.

Spens, J., Evans, A. R., Halfmaerten, D., Knudsen, S. W., Sengupta, M. E., Mak, S. S. T., Sigsgaard, E. E. \& Hellström, M. (2016). Comparison of capture and storage methods for aqueous macrobial eDNA using an optimized extraction protocol: advantage of enclosed filter. Methods in Ecology and Evolution 8, 635-645.

Spitzen-van der Sluijs, A., Stark, T., DeJean, T., Verbrugghe, E., Herder, J., Gilbert, M., Janse, J., Martel, A., Pasmans, F. \& Valentini, A. (2020). Using environmental DNA for detection of Batrachochytrium salamandrivorans in natural water. Environmental DNA 0, 1-7.

Strickler, K. M., Fremier, A. K. \& Goldberg, C. S. (2015). Quantifying effects of UV-B, temperature, and $\mathrm{pH}$ on eDNA degradation in aquatic microcosms. Biological Conservation 183, 85-92.

Takahara, T., Iwai, N., Yasumiba, K. \& Igawa, T. (2019). Comparison of the detection of 3 endangered frog species by edna and acoustic surveys across 3 seasons. Freshwater Science 39, 19-27.

Thomsen, P. F., Kielgast, J., Iversen, L., Wiuf, C., Rasmussen, M., Gilbert, M. T. P., Orlando, L. \& Willerslev, E. (2012). Monitoring endangered freshwater biodiversity using environmental DNA. Molecular Ecology 21, 2565-2573.

Torresdal, J. D., Farrell, A. D. \& Goldberg, C. S. (2017). Environmental DNA detection of the golden tree frog (Phytotriades auratus) in bromeliads. PLOS ONE 12, e0168787.

Turner, C. R., Uy, K. L. \& Everhart, R. C. (2015). Fish environmental DNA is more concentrated in aquatic sediments than surface water. Biological Conservation 183, 93-102.

Untergasser, A., Cutcutache, I., Koressaar, T., Ye, J., Faircloth, B. C., Remm, M. \& Rozen, S. G. (2012). Primer3-new capabilities and interfaces. Nucleic Acids Research 40(15), e115.

Valentin, R. E., Fonseca, D. M., Gable, S., Kyle, K. E., Hamilton, G. C., Nielsen, A. L. \& Lockwood, J. L. (2020). Moving eDNA 
surveys onto land: Strategies for active eDNA aggregation to detect invasive forest insects. Molecular Ecology Resources 20, 746-755.

Zinger, L., Donald, J., Brosse, S., Gonzalez, M. A., Iribar, A., Leroy, C., Murienne, J., Orivel, J., Schimann, H., Taberlet, P. \& Lopes, C. M. (2020). Advances and prospects of environmental DNA in neotropical rainforests. Advances in Ecological Research 62, 331-373.
Accepted: 28 September 2020 